University of Nebraska - Lincoln

DigitalCommons@University of Nebraska - Lincoln

Industrial and Management Systems

Industrial and Management Systems

Engineering Faculty Publications

Engineering

7-6-2007

\title{
Understanding Sub-20 nm Breakdown Behavior of Liquid Dielectrics
}

Kumar R. Virwani

University of Arkansas Community College - Hope

Ajay P. Malshe

University of Arkansas, apm2@engr.uark.edu

Kamlakar P. Rajurkar

University of Nebraska-Lincoln, krajurkar1@unl.edu

Follow this and additional works at: https://digitalcommons.unl.edu/imsefacpub

Part of the Operations Research, Systems Engineering and Industrial Engineering Commons

Virwani, Kumar R.; Malshe, Ajay P.; and Rajurkar, Kamlakar P., "Understanding Sub-20 nm Breakdown Behavior of Liquid Dielectrics" (2007). Industrial and Management Systems Engineering Faculty Publications. 16.

https://digitalcommons.unl.edu/imsefacpub/16

This Article is brought to you for free and open access by the Industrial and Management Systems Engineering at DigitalCommons@University of Nebraska - Lincoln. It has been accepted for inclusion in Industrial and Management Systems Engineering Faculty Publications by an authorized administrator of DigitalCommons@University of Nebraska - Lincoln. 


\title{
Understanding Sub-20 nm Breakdown Behavior of Liquid Dielectrics
}

\author{
Kumar R. Virwani, ${ }^{1}$ Ajay P. Malshe, ${ }^{2, *}$ and Kamlakar P. Rajurkar ${ }^{3}$ \\ ${ }^{1}$ Department of Microelectronics Photonics, University of Arkansas, Fayetteville, Arkansas 72701, USA \\ ${ }^{2}$ Department of Mechanical Engineering, University of Arkansas, Fayetteville, Arkansas 72701, USA \\ ${ }^{3}$ Department of Industrial and Management Systems Engineering, University of Nebraska, Lincoln, Nebraska 68588, USA
}

(Received 30 May 2006; published 6 July 2007)

\begin{abstract}
Nanoscale confinement of dielectric molecules is expected to influence their breakdown mechanism in applications such as nanoprobe based machining, molecular electronics, and other related technologies. This Letter presents the first experimental study of the breakdown of nonpolar, nonthiolated liquid dielectrics in the nanometer regime and develops a field emission assisted avalanche based approach to model such behavior. The studies show that dielectric breakdown in the sub- $20 \mathrm{~nm}$ regime is independent of the cathode materials and is dominated by the electron emission and atomic cluster migration due to the "sub-20 nm scale confinement of the liquid dielectric."
\end{abstract}

DOI: 10.1103/PhysRevLett.99.017601

Fabrication of features such as nanopores for DNA detection devices, through nanovias for $Z$ axes interconnects, nanojets for controlled drug release, and nozzles for nanofluidic devices in nonsilicon difficult to machine materials is of immense technological importance. For difficult-to-machine materials, processes based upon the breakdown (BD) of liquid dielectrics have traditionally been used for controlled macroscale [1] and microscale [2] material removal in electric discharge machining (EDM). A recent work by the authors of this Letter [3] demonstrated that controlled electromachining in dielectrics confined in nanometer spaces can also be used to perform material removal at the sub- $20 \mathrm{~nm}$ scale regime in atomically flat gold; a process referred to from here on as nanoelectromachining (nano-EM). Because of small (0.5$20 \mathrm{~nm}$ ) cathode-anode gaps, the electric fields generated in nano-EM are intense $\left(\sim 10^{7}-10^{9} \mathrm{~V} / \mathrm{m}\right)$, even for voltages in the region of $100 \mathrm{mV}$ to $30 \mathrm{~V}$. In bulk dielectrics [4], field strengths of about $10^{6}-10^{7} \mathrm{~V} / \mathrm{m}$ are sufficient to cause the BD of dielectrics with the Townsend avalanche as the dominant mechanism. However, nanoscale imaging of dielectric molecules like alkanes is routinely performed using a scanning tunneling microscope [5], where the electric field strength is about $10^{8} \mathrm{~V} / \mathrm{m}$ under typical imaging conditions. Such high field strength does not result in the $\mathrm{BD}$ of dielectric molecules [6], proving that $\mathrm{BD}$ processes at the nanoscale are fundamentally different from BD processes at the macroscale. Thus, the nanoscale dielectric molecules may be preferentially oriented [7] in certain directions due to spatial confinement and chemicalpotential boundary conditions. This study attempts to understand, for the first time, dc breakdown behavior of nonthiolated normal alkanes across sub-20 nm gaps.

The first experiments on electrical discharges across very small gaps were performed in the year 1900 by Earhart [8] and later by Hobbs [9]. Their studies showed that the BD of air occurred below the Paschen minimum for air, which at that time was believed to be about $340 \mathrm{~V}$, and that the gaseous BD in the cathode-anode gaps up to
PACS numbers: $77.22 . J p, 52.25 . \mathrm{Mq}, 52.80 . \mathrm{Wq}$

3 times the wavelength of sodium was independent of the gas [9]. In dielectric liquids, the BD processes result in the formation of streamers [10] from both electrodes initiating from sharp points. Schmidt [11] discussed the roles of the electrons, the holes, and the ions in the $\mathrm{BD}$ of dielectric liquids. A recent review [12] of the $\mathrm{BD}$ in $\mathrm{SiO}_{2}$ reported that in thick $(\sim 50 \mathrm{~nm})$ oxides, cracks propagate across the entire film and for very thin $(\sim 1 \mathrm{~nm})$ oxides a singular feature is generated in the film upon BD. In an allied and emerging field of molecular and organic electronics [13], understanding dielectric properties of very thin layers would play a critical role in the next generation electronic devices. Most probe-based applications which utilize the cathode point tip and anode plane configurations for experimental analysis could benefit from this study. Hence, a fundamental understanding of the dielectric breakdown behavior across sub-20 $\mathrm{nm}$ cathode-anode gaps forms the basis of this Letter.

A scanning tunneling microscope (STM) from Digital Instruments with a NanoScope $E$ controller allowed the physical positioning of the cathode point and also topographical scanning of the anode plane surface after BD. An Agilent 3632A dc generator was used for bias control using LABVIEW $^{\mathrm{TM}}$ software. Etched tungsten and platinum:iridium (80:20) alloys were chosen as cathode materials, and atomically flat hydrogen flame annealed gold acted as an anode material. Electrochemical etching in $2 \mathrm{M}$ $\mathrm{NaOH}$ was used to fabricate consistent geometry tungsten cathodes with an end radius of $35 \mathrm{~nm}(+/-10 \mathrm{~nm})$ [14]. Electrochemically etched Pt-Ir cathodes were obtained from Molecular Imaging Corporation and had an end radius of about $35 \mathrm{~nm}$. Current-displacement spectroscopy curves were used to quantify the quality of the cathodes [14]. The low voltage electric field response from the cathodes was analyzed to ensure the similarity of the cathode end radii and the apex shapes [14]. For the experiment, the gold samples were mounted with silver paste on to the sample holders and their conductivities verified. The dielectric liquid, $n$-decane [15] was introduced in the 
sample holder and a topographic scan obtained at $500 \mathrm{pA}$ tunneling current and $100 \mathrm{mV}$ bias voltage. To perform the BD studies, the cathode was moved away from the anode by changing the $Z$-piezo voltages and was allowed about $2 \mathrm{sec}$ to settle. Next, the tunneling current feedback was turned off. The voltage was increased in steps of $1 \mathrm{~V}$ each for $60 \mathrm{~ms}$ which was the maximum data transfer speed of the communication bus. The tunneling current feedback was enabled at the end of the breakdown cycle in order to avoid the crashing of the cathode into the anode.

Figure 1 shows the current-voltage BD characteristics for a gold anode and an etched tungsten cathode in $n$-decane across a $3 \mathrm{~nm}$ gap. The current measured was zero until the applied voltage was about $4.8 \mathrm{~V}$ and at this point the current started to increase with the voltage and at $6 \mathrm{~V}$ dielectric $\mathrm{BD}$ occurred. In order to plot the Paschen curves for $n$-decane, the current-voltage BD curves were collected at the cathode-anode separations of 3, 5, 8, 10, $13,15,18$, and $20 \mathrm{~nm}$. Within experimental error (10\%), the BD behavior of $n$-decane was measured to be the same with $\mathrm{W}$ and Pt-Ir in the sub-20 $\mathrm{nm}$ region and increased linearly with the cathode-anode separation as shown in Fig. 2. A comparison of the scanning electron micrographs (Fig. 3) of the cathode before and after the BD indicates that the cathode had vaporized to a near flat end [shaded region in Fig. 3(a)] and its diameter had increased by about $15 \%$ post-BD. Hence, for each data point a new etched cathode was used. The inset in Fig. 3(b) shows an SEM micrograph of the cathode apex for the BD across a $15 \mathrm{~nm}$ gap, suggesting the melting of the apex.

The measured BD field strength (Fig. 2) of $1 \times$ $10^{9} \mathrm{~V} / \mathrm{m}$ is in good order of magnitude agreement with the BD field strength for vacuum gaps [16] across nanometer dimensions. The BD field strength was found to be independent of the cathode material for $\mathrm{W}$ and Pt-Ir alloy (work functions 5.5 and $4.5 \mathrm{eV}$, respectively), unlike at the

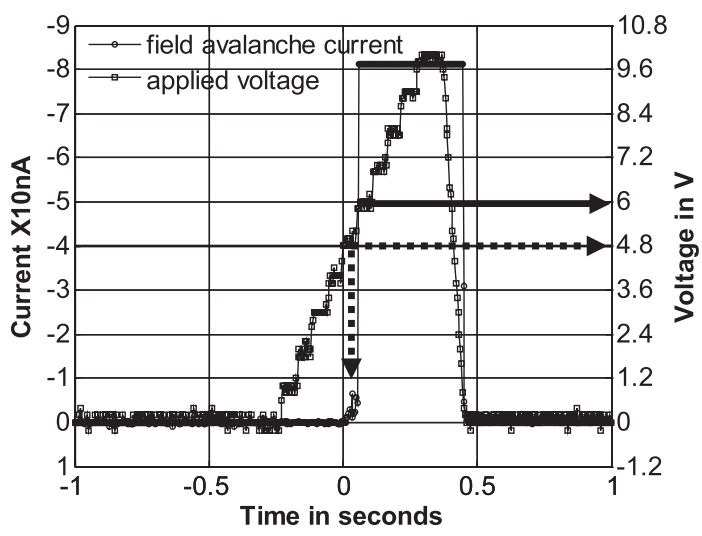

FIG. 1. The breakdown characteristics of $n$-decane across $3 \mathrm{~nm}$ cathode-anode gap. Instabilities in the current-voltage curve start at a gap potential of about $4.8 \mathrm{~V}$ (dashed arrows), and at about $6 \mathrm{~V}$ (solid arrow) complete dielectric breakdown was measured. The recovery of dielectric strength was not complete until about $25-30 \mathrm{~ms}$ from the time voltage dropped below the initial instability voltage $(\sim 4.8 \mathrm{~V})$. macro and microscales [17]. This phenomenon suggests that the BD is related to the confinement of the molecular dielectric in nanoscale gaps and the net applied electric field stress. The authors present the following model to understand the breakdown behavior in the nanometer regime. Upon increasing the applied voltage to cause field strength of about $0.9 \mathrm{~V} / \mathrm{nm}$, the gold atoms from the atomic steps [18] and tungsten atoms from the tip [19] start to migrate into the cathode-anode gap. Because of the high field strength, positive space charges form at the gold anode [20]. At the nanoscale, the primary energy input into the gap is from the field emission process resulting in an avalanche breakdown. The process hereafter is referred to as a "field avalanche." Even though the breakdown field predicted by the existing spark discharge models [4] is about $7.5 \times 10^{7} \mathrm{~V} / \mathrm{m}$, the experimentally measured field is about $1 \times 10^{9} \mathrm{~V} / \mathrm{m}$. These electric field strengths are in good agreement with previous studies where emission has been measured from the tungsten tips coated with a thin layer of epoxy [21]. The nanoconfinement of the dielectric liquid molecular medium masks the effect of the work function of the electrode materials for the $\mathrm{W}$ and Pt-Ir alloy.

The electron emission deposited thermal energy in the gap resulting in a phase change of $n$-decane molecules from a confined-quasi-organized molecular medium to a highly activated-disordered-ionized molecular medium. Upon dielectric breakdown, the gap acted as a short in the electrical circuit. The current at BD was limited by the power supply to $100 \mathrm{~mA}$. This current flowing through a cathode shank diameter of about $100 \mathrm{~nm}$ resulted in a current density of $1.3 \times 10^{13} \mathrm{~A} / \mathrm{m}^{2}$ causing heating, melting, and eventual vaporization [22] of tungsten [Fig. 3(a)]. Thus, upon breakdown the gap consisted of hydrocarbon, tungsten, and gold atomic and molecular vapors. This

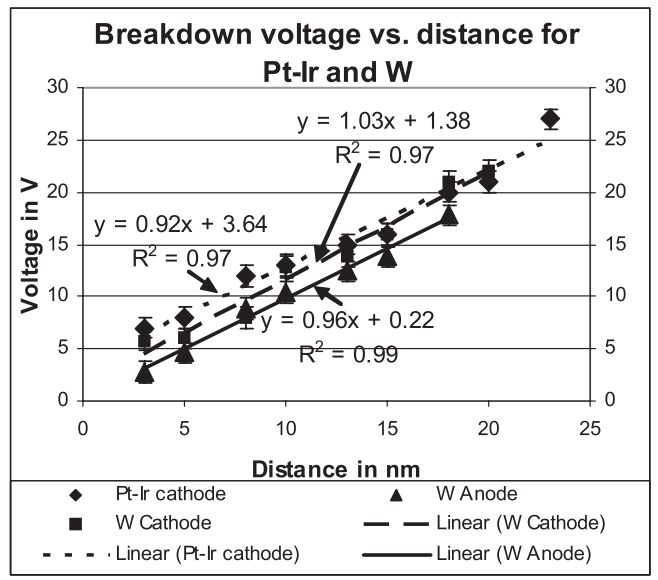

FIG. 2. Paschen curves for the breakdown of $n$-decane across increasing gaps with a flat gold anode and etched W and Pt-Ir cathodes and for flat gold cathode and etched $\mathrm{W}$ anode. The linearly increasing electric field strength required for breakdown was measured to be about $1 \times 10^{9} \mathrm{~V} / \mathrm{m}$, irrespective of the voltage polarity. 


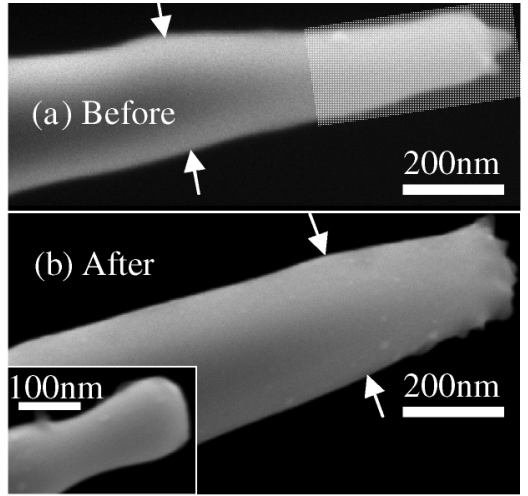

FIG. 3. SEM micrographs of a tungsten cathode before and after breakdown. The end radius of the cathode increased from about $35 \mathrm{~nm}$ before breakdown (a) to a near flat end after breakdown (b) and the shank diameter increased by $15 \%$. The inset in (b) shows the melting event captured for breakdown at $15 \mathrm{~nm}$ gap.

"nanoexplosion" of the vapor at BD exerted an upward pressure on the molten tungsten [Fig. 3(b), inset] cathode causing a $15 \%$ increase in the shank diameter. In the future, the authors plan to use current limiting diodes to prevent damage to the cathode tips.

When the applied voltage was removed, the gap did not recover its strength at the $\mathrm{BD}$ voltage. Instead the current continued to flow even when the voltage dropped to about $500 \mathrm{mV}$. The gap would recover its strength when the fresh dielectric molecules replace the vapors in the gap. In the absence of any external dielectric flow, unlike the EDM process, the motion of the molecules is driven by the diffusion process due to the concentration gradient between the expanding gas and the dielectric molecules. Thus, the nanoscale chemoelectromechanical space confinement of the dielectric may also be responsible for the delayed recovery of gap strength [23]. A recent study [7] showed that the liquid molecules form condensates and demonstrate solidlike behavior in the presence of a single interface. The gold anode provided such an interface in the current study. Hence, for processes based upon nanoconfined liquid dielectrics, one may have to design to account for the influence of the flow characteristics and the molecular dielectric replenishment dynamics.

The study of the breakdown mechanism revealed that the current-voltage curves followed a behavior similar to the macroscale process of electric discharge machining, although at field strengths, which were 17 times stronger. The overall current-voltage behavior in Fig. 1 could be divided into distinct regions such as prebreakdown (voltage $<6 \mathrm{~V}$ ), breakdown (between breakdown voltage, $6 \mathrm{~V}$ and where the pulse was shut down, $100 \mathrm{mV}$ bias was maintained as STM feedback voltage), and postbreakdown. Similar regions are observed in the macroscale breakdown behavior. Hence, based on the research presented in this Letter, the authors would like to identify the
nano-EM process as being the nanoscale electric discharge machining (nano-EDM) process.

A model based on a field emission assisted avalanche was developed to predict the breakdown field strengths. In order to understand the effects of dielectric ionization, Townsend primary ionization coefficients and the current enhancement ratios were estimated from the equations $\frac{\alpha}{p_{0}}=A_{0} \exp \left(-\frac{B_{0}}{E / p_{0}}\right)$ and $E_{T}=\frac{I}{I_{0}}=\frac{e^{\alpha d}}{1-\gamma\left(e^{\alpha d}-1\right)}$ where $\alpha$ and $\gamma$ are the Townsend primary and secondary ionization coefficients, $p_{0}$ is the pressure of the dielectric, $A_{0}$ and $B_{0}$ are constants, $E$ is the applied electric field, $E_{T}$ is the Townsend current enhancement ratio, and $d$ is the gap. There is "no analytical expression for the evaluation of $\alpha$ in the case of liquids" [24]. Hence, to determine $\alpha$, the behavior of the $n$-decane liquid was approximated to the behavior of $n$-decane gas under high pressure [25]. Based upon previous studies [25] performed on propane, a homologue of the alkane series, a pressure of $12 \mathrm{MPa}$ was chosen for the analysis. The coefficients $A_{0}, B_{0}$, and $\gamma$ for gaseous $n$-decane state were estimated by extrapolating data for normal alkanes [25] and their values were $47.55 \mathrm{~cm}^{-1} \mathrm{~mm}^{-1}, 760 \mathrm{~V} \mathrm{~cm}^{-1} \mathrm{~mm}$, and $10^{-8}$, respectively. The Townsend current multiplication ratio $E_{T}$ increases from 0.36 at $3 \mathrm{~nm}$ to 6.5 at $18 \mathrm{~nm}$, which is the range of the experimental data. However, the experimental current rises from $50 \mathrm{pA}$ to about $80 \mathrm{nA}$ at breakdown, a ratio of about 1600 . Hence, the Townsend enhancement alone could not account for the measured breakdown currents.

Current in the gap collectively due to field avalanche and Townsend enhancement can be estimated from the product of Fowler-Nordheim current [26] and the Townsend enhancement ratio as

$$
\begin{aligned}
I= & E_{T}\left(\frac{6.2 \times 10^{-6}}{\phi+\mu}\right)\left(\frac{\mu}{\phi}\right)^{1 / 2}\left(\frac{V}{d}\right)^{2} \\
& \times \exp \left(-6.8 \times 10^{7} \times \phi^{1.5} \times \frac{d}{V}\right),
\end{aligned}
$$

where $V$ is the applied voltage, $\phi$ is the work function, $\mu$ is the Fermi energy level, and $I$ is the gap current. The numerical values of $5.8 \mathrm{eV}, 4.55 \mathrm{eV}$, and $35 \mathrm{~nm}$ were used for the Fermi energy, work function, and end radius, respectively. For experimentally measured distances, the values for $V$ which result in a current of $80 \mathrm{nA}$ (measured current at the BD) across the gap were predicted. An average value of $5 \times 10^{9} \mathrm{~V} / \mathrm{m}$ was obtained for the breakdown field, shown for the case of the $3 \mathrm{~nm}$ gap in Fig. 4 . This value is in close agreement with the experimentally obtained value of $1 \times 10^{9} \mathrm{~V} / \mathrm{m}$. Macroscale models predict a BD field strength of $7.5 \times 10^{7} \mathrm{~V} / \mathrm{m}$. In comparison, the field avalanche model predicts a breakdown strength of $5 \times 10^{9} \mathrm{~V} / \mathrm{m}$. This implies that a nanoconfined dielectric requires about 66 times higher field for breakdown when compared with a macroconfined dielectric. The authors believe that the 5 times increase in the field, between the field avalanche model and the measured experimental 


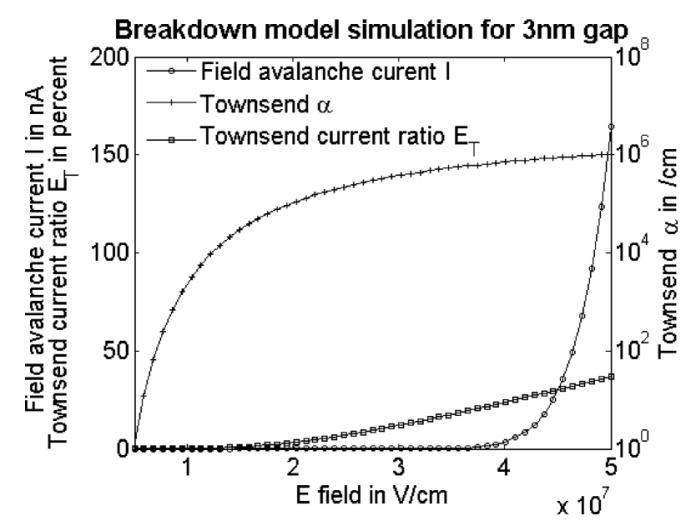

FIG. 4. The simulation of the variation of the Townsend $\alpha$, the Townsend current ratio $E_{T}$, and the field avalanche current $I$ with the applied field for a $3 \mathrm{~nm}$ gap. The Townsend enhancement alone is not sufficient to explain the high current of tens of nanoamperes at breakdown.

value is a result of the space charge enhancement in the gap. It may be noted that for very large values of $\mathrm{V} / \mathrm{d}$, and, in particular, when " $\mathrm{d}$ " is less than the end radius of the electrode, the energy available for the breakdown process is dominated only by the value of the field strength and not the work function or the polarity of the cathode material as measured experimentally. In a recent study [19], the authors provide experimental evidence that in addition to electron emission, tungsten atom migration under the influence of a strong electric field also results in lowering of the gap strength. Further modeling which includes the effects of atomic migration in the modification of gap strength is required to understand the detailed mechanism of the phenomena. It is expected that with increasing cathode-anode separations the work functions of the electrode materials would start to play a role in the BD process.

In conclusion, breakdown studies were performed on pure $n$-decane liquid molecules confined in the sub$20 \mathrm{~nm}$ gaps between atomically flat gold and $\sim 35 \mathrm{~nm}$ end radius $\mathrm{W}$ and Pt-Ir electrodes. A distinctly different breakdown mechanism due to the field emission assisted avalanche in nanoconfined liquid dielectrics is illustrated by both the experimental observations and the developed model. The nanoscale confinement masks the effects of the work function of the cathode material. The breakdown characteristics were found to be independent of the cathode material in the sub-20 $\mathrm{nm}$ region. The recovery of the dielectric strength after breakdown showed a time lag after cessation of the voltage, suggesting molecular diffusion as a mechanism for dielectric replenishment. A process model developed for nanometer confined dielectric liquids suggests that the required electric field is about 66 times stronger in comparison with the macroscale dielectrics. For nanoscale machining based on dielectric breakdown, the overall response of the system is expected to be dominated by the recovery time of the dielectrics in confined spaces and on the behavior of the anode-dielectric-cathode interface.

Authors acknowledge the financial support from the National Science Foundation (NSF) for this research via CMMI (Grant No. 0423698).

*Corresponding author. apm2@engr.uark.edu

[1] M. Kunieda, B. Lauwers, K. P. Rajurkar, and B. M. Schumacher, CIRP Annals 54, 599 (2005).

[2] K. P. Rajurkar and Z. Y. Yu, CIRP Annals 49, 127 (2000).

[3] A.P. Malshe, K. Virwani, K.P. Rajurkar, and D. Deshpande, CIRP Annals 54, 175 (2005).

[4] A. Cheglokov, M. Noskov, V. Lopatin, and A. Shapovalov, 5th Korea-Russia International Symposium on Science and Technology. Proceedings. KORUS 2001 (2001) p. 224.

[5] A. Marchenko, J. Cousty, and L. Pham Van, Langmuir 18, 1171 (2002).

[6] M. Rolandi, K. Scott, E. G. Wilson, and F. C. Meldrum, J. Appl. Phys. 89, 1588 (2001).

[7] K. B. Jinesh and J. W. M. Frenken, Phys. Rev. Lett. 96, 166103 (2006).

[8] R. F. Earhart, Philos. Mag. 1, 147 (1901).

[9] G. M. Hobbs, Philos. Mag. 10, 617 (1905).

[10] E. O. Forster, IEEE Trans. Electr. Insul. 25, 45 (1990).

[11] W. F. Schmidt, IEEE Trans. Electr. Insul., 26, 560, (1991).

[12] S. Lombardo, J.H. Stathis, B.P. Linder, K. L. Pey, F. Palumbo, and Chih Hang Tung, J. Appl. Phys. 98, 121301 (2005).

[13] M. A. Reed, Nat. Mater. 3, 286 (2004).

[14] K. R. Virwani, K.P. Rajurkar, and A.P. Malshe, J. Nanoeng. Nanosyst. 220, 1 (2006).

[15] P. C. Landolt-Börnstein, Static Dielectric Constants of Pure Liquids and Binary Liquid Mixtures, edited by C. Wohlfarth and O. Madelung (Springer Verlag, Berlin, 1991), p. 521.

[16] H. Maruo, Y. Hirata, and K. Ozaki, Yosetsu Gakkai Ronbunshu: Quart. J. Jpn. Welding Soc. 12, 477 (1994).

[17] R. M. Schaffert, Electrophotography (Wiley International, New York, 1975).

[18] T. M. Mayer, J. E. Houston, G. E. Franklin, A. A. Erchak, and T. A. Michalske, J. Appl. Phys. 85, 8170 (1999).

[19] K. R. Virwani, K. P. Rajurkar, and A.P. Malshe, CIRP Annals (to be published).

[20] W. S. Boyle, P. Kisliuk, and L. H. Germer, J. Appl. Phys. 26, 720 (1955).

[21] R. V. Latham and M. S. J. Mousa, J. Phys. D 19, 699 (1986).

[22] N. I. Kuskova, S. I. Tkachenko, and S. V. Koval, Int. J. Thermophys. 19, 341 (1998).

[23] K. R. Virwani, K. P. Rajurkar, and A.P. Malshe (unpublished).

[24] M. Haidara and A. Denat, IEEE Trans. Electr. Insul. 26, 592 (1991).

[25] A. E. D. Heylen, Proc. R. Soc. A 456, 3005 (2000).

[26] R. Gomer, Field Emission and Field Ionization (Springer, New York, 2004). 